\title{
Interlaboratory Comparison of Immunohistochemical Testing for Equivocal Her2: Results of the 2014-2017 Center-Southern Italy HER2 Survey
}

\author{
Ilaria Pennacchia ${ }^{1}$, Armando Orlandi $^{3}$, Marcella Mottolese ${ }^{2}$, Francesco Pierconti $^{1}$, Arnaldo Carbone ${ }^{1}$ \\ and Vincenzo Arena ${ }^{1 *}$
}

${ }^{1}$ IRCCS Fondazione Policlinico Universitario A Gemelli, Department of woman and child health and public health -Pathology Area -Università Cattolica del Sacro Cuore -Istituto di Anatomia Patologica, Roma

${ }_{2}^{2}$ Istituto di Anatomia Patologica- Istituto Nazionale Tumori Regina Elena-Roma

${ }^{3}$ Istituto di Oncologia Medica-IRCCS Fondazione Policlinico Universitario A. Gemelli Roma

*Corresponding author: Vincenzo Arena, Istituto di Anatomia Patologica Università Cattolica del Sacro Cuore L. go F Vito

100168- Roma, Italy

\section{ARTICLE INFO}

Received: 豐 August 02, 2019

Published: 幽 August 09, 2019

Citation: Ilaria Pennacchia, Armando Orlandi, Marcella Mottolese, Francesco Pierconti, Arnaldo Carbone, Vincenzo Arena. Interlaboratory Comparison of Immunohistochemical Testing for Equivocal Her2: Results of the 20142017 Center-Southern Italy HER2 Survey. Biomed J Sci \& Tech Res 20(3)2019. BJSTR. MS.ID.003454.

Keywords: HER2 Immunohistochemistry; Quality Control; Interlaboratory Reproducibility

\section{ABSTRACT}

Correct assessment of HER2 status is essential for managing patients with invasive breast cancer, but few data are available on the accuracy of laboratories performing HER2 testing by immunohistochemistry (IHC).

Objective: To review the results of the 2014-2017 Center-Southern Italy HER2 Survey. DESIGN: The HER2 survey was designed for laboratories performing immunohistochemical staining and interpretation for HER2 and used routine slides of different HER "2+" invasive breast carcinomas. All cases were also analyzed by DDSISH. All participants performed immunostaining for HER2 using the laboratory's standard procedures. Each participant center interpreted the stained slides and returned results to the coordinator center for data analysis. This survey included 41 laboratories from Center-South Italy.

Results: 28/41 (68\%) centers carried out "correct" HER2 IHC determinations. On the contrary 13/41 (32\%) centers obtained concordance values lower than $90 \%$.

Conclusion: Performance of laboratories dealing with HER2 IHC determinations in breast invasive carcinoma in this survey was good.

\section{Introduction}

In recent years many efforts have been made aiming to ensure the efficiency of each pathology lab in HER2 IHC determination in breast and in gastric cancer [1-6]. The most important antibody manufacturers have certainly tried to provide excellent quality standards and the specificity/sensitivity results of the single antibody clones are well known. However, despite all the efforts made by the scientific community, many years after the discovery of the role of HER2 in breast cancer, and 15 years after the clinical use of trastuzumab in patients with overexpression and/ or amplification of HER2, it frequently happens that oncologists request a second opinion for Her2-status determinations on coreneedle-biopsies or surgical specimens.
The reason why this happens is usually because the reviewing pathologist works in a hospital with a larger number of cases of breast cancer or the initial results appear discordant to the oncologist. In our experience second opinions are most often requested on HER2 status determinations performed by small or private laboratories. Being aware that each laboratory (regardless of its annual number of cases and /or geographical location) works to the best of its ability, with this study we wanted to "virtually" recreate what happens in our daily practice to evaluate if the need for a second opinion of HER2 status is really determined by the poor performance of peripheral laboratories. 


\section{Materials and Methods}

41 Central-Southern Italy pathology laboratories joined the project, the one in Policlinico A. Gemelli being appointed as the reference laboratory. All the participating centers were divided into homogeneous groups by ensuring that each had at least one center with an average number of 150 cases per year. Each center was asked to select two cases with a given score $(2+$ amplified and 2+ not amplified sec. 2013 ASCO/CAP guidelines6) and to prepare three blank sections to be sent to the other members of the group. Three additional sections were required to be sent to the coordinating center. The project was launched in November 2014 and was completed by May 2017. Slides were dispatched by ordinary mail or courier. Each center was asked to stain the slides received and to assign a score using both the scoring system applied before the release of the ASCO/CAP Guidelines6, and the new criteria contained in them. Each center was also asked to complete a first questionnaire asking for information such as laboratory methods used for the immunohistochemical determination of HER2 (antibody type, platform, ISH).A second questionnaire was administered asking for the number of each HER2 score $(0,1+, 2+, 3+$, ISH Amplified/Not amplified) observed respectively in the 12 months before and after the publication of the ASCO/CAP Guidelines.

\section{Results}

Most slides arrived at destination undamaged, therefore each center could perform the staining and evaluate the HER2 status. Each center received the slides from the other centers belonging to the same group. No mandatory instructions were given as to how to do the staining runs, that is there was no obligation to make a single staining run on all received slides. As recommended, each lab carried out the staining as it could, with its own timing and without interfering with the routine diagnostic activities carried out by the lab. We emphasize this point because this is what happens in real lab-life when "external" cases are sent to counseling (not all arrive at the same time, and not all are immune stained in the same run).

The slides sent to the Policlinico Gemelli laboratory (referring center) were immune stained with 4B5 antibody, while for determining the HER2 amplification status the DDSISH method was adopted. Each center was requested to send the results via email to the coordinating centers for the data analysis. Importantly, this study was given a practical profile, especially as regards the concordance analyses given that the aim was to evaluate accuracy of HER2 status HER2 determination only in relation to the therapeutic approach. If a case sent as $2+$ not amplified was scored $1+$ or 0 by the other centers belonging to the group, no consideration was given to possible immune stain discordances (since the case was not eligible for therapy).It is known that this approach may annoy the "purists" of immunohistochemical determinations, however, the Her2 status of breast cancer represents undoubtedly a dichotomous (negative/ positive) category in the oncologist's decision making process.
During the central review of cases the primary goal purpose was to find out possible discrepancies between the scores assigned by the participating centers and the results obtained by repeating tests at the referring center and to see whether these discordances could impact therapeutic plans. This situation occurred only in one case that had been sent with a score of " $2+$ Not Amplified" and finally turned out to be a $2+$ Amplified score by SISH. Clinicians were promptly informed, and the patient was enrolled for treatment with TrastuzumAb without the initial wrong determination being harmful to the patient's treatment plan. In the light of what above said, and with respect to the results generated by the data analysis we should underline that regardless of the antibody that was used, 28/41 centers carried out "correct" HER2 determinations. On the contrary 13/41 centers have obtained concordance values lower than $90 \%$. For each center that did not reach acceptable levels of concordance with the reference scores we analyzed the results identifying the specific problems.

We believe that this approach adds value to this interregional analysis since the direct knowledge of participants and the easy of communication among them could eliminate all barriers. We all worked on an equal footing with the aim of improving our own performance and consequently the quality of the HER2 immunohistochemical determination without the need to seek a "primus inter pares". Of all laboratories getting low performance values we will discuss only the one with the lowest value. From our data analysis it came out that of the 10 slides that were to be scored, the laboratory in question stained and correctly identified the HER2 status only in 6 cases. When interviewing the lab pathologists, we learnt that they were aware of the low performance in HER2 IHC determination and that they have already informed the head physician as well as to the administrative office of the problem.

However, due to a number of reasons, among which internal bureaucratic delays, there had been no possibility to change the existing antibody supply with another one with a more proven reliability. The analysis of concordance performed on the other participating centers enabled us to point out the underlying reasons (analytical, pre-analytical or post-analytical) for the mistakes that were found. As mentioned above, each center was also asked to provide the overall positivity and negativity HER2 data in order to assess whether the 2013 ASCO/CAP guidelines had a real impact on the daily lab procedures. The review period went from November 2012 to October 2015 and a total of 16231HER2 status determinations were analyzed (Table 1). A further demonstration that the goal of the 2013 ASCO/CAP guidelines had been achieved was given by the fact that during the period following the release of the new guidelines, the overall number of patients eligible for therapy $[(2+\mathrm{Amp})+(3+)]$ increased from 15 to 18 percent (Figure 1 and Table 1). From a purely numerical point of view as many as 204 extra patients could benefit from a targeted therapeutic treatment (with Trastuzumab) in the Italian regions that were enrolled in this survey. 


\section{SURVEY HER2 2013-2016}

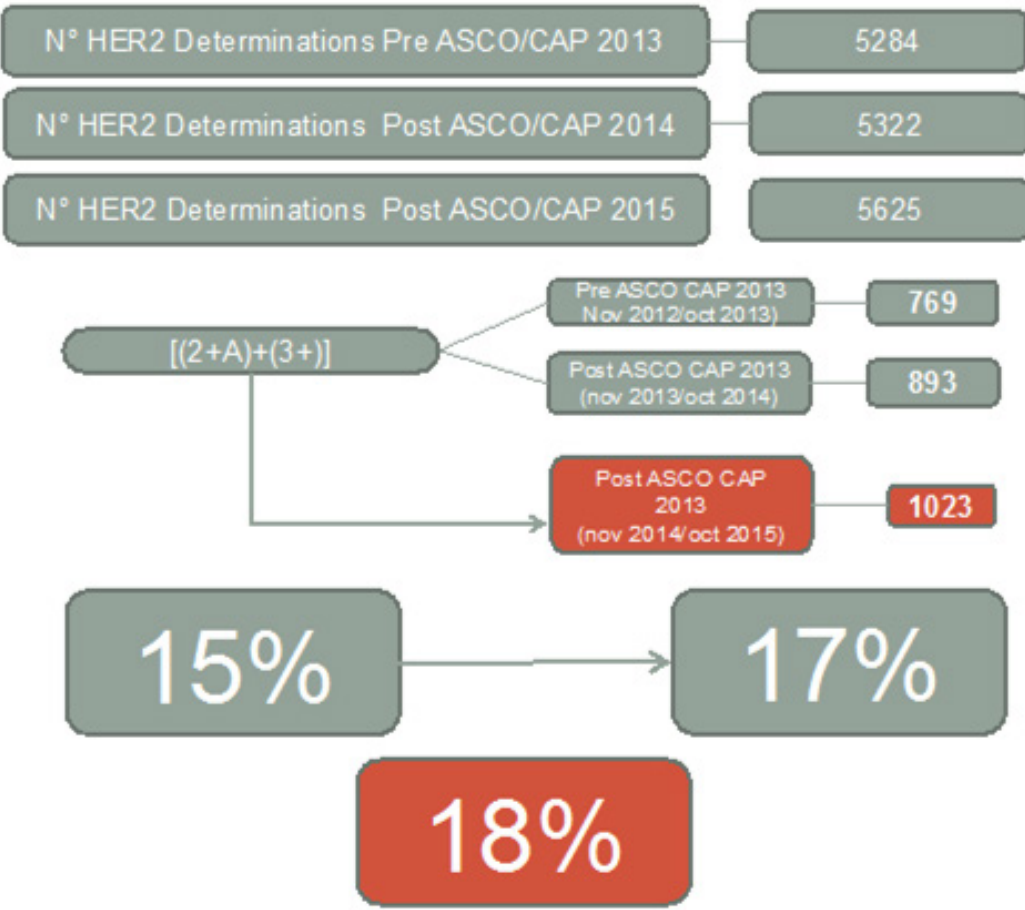

Figure 1.

Table 1.

\begin{tabular}{|c|c|c|c|}
\hline Score & Nov2012/Oct2013 & Nov2013/Oct2014 & Nov2014/Oct2015 \\
\hline 0 & $42 \%$ & $38 \%$ & $37 \%$ \\
\hline $1+$ & $31 \%$ & $30 \%$ & $29 \%$ \\
\hline $2+$ & $17 \%$ & $21 \%$ & $21 \%$ \\
\hline $3+$ & $10 \%$ & $12 \%$ & $12 \%$ \\
\hline Score & Nov2012/0ct2013 & Nov2013/0ct2014 & Nov2014/0ct2015 \\
\hline Amplified & $24 \%$ & $24 \%$ & $26 \%$ \\
\hline
\end{tabular}

\section{Conclusion}

After the release of the ASCO/CAP 2013 guidelines there have been many reports aiming at highlighting the apparent "inaccuracies" and contributing to properly define the various diagnostic categories. It is well known that the goal of the new ASCO/CAP guidelines was not to "lose" patients who are potentially eligible for treatment with Trastuzumab by lowering the positivity thresholds. Based on this analysis conducted on most labs in Southern and Central Italy, we can conclude that if the goal of the 2013 ASCO/ CAP guidelines was to be able to recruit new patients, at least the Italian centers that were surveyed achieved it. We mean to extend this experience to other centers in the near future, as it allows to attain more and more the "precision pathology" goal, which does not mean not making any mistake at all, but rather trying to minimize the number of possible errors for the sake of patients.

\section{Acknowledgement}

We thank all centers that participated in the study and that are part of the Interregional Survey Group.

\section{References}

1. Press OA, Guzman R, Cervantes M Santiago A, Press MF (2014) Characterization of HER2 status by fluorescence in situ hybridization (FISH) and immunohistochemistry (IHC). Methods Mol Biol 1180: 181207.

2. Sheffield BS, Garratt J, Kalloger SE Li-Chang HH, Torlakovic EE, et al. (2014) HER2/neu testing in gastric cancer by immunohistochemistry: assessment of interlaboratory variation. Arch Pathol Lab Med 138(11): 1495-1502.

3. Bianchi S, Caini S, Paglierani M, Saieva C, Vezzosi V, et al. (2015) Accuracy and Reproducibility of HER2 Status in Breast Cancer Using Immunohistochemistry: A Quality Control Study in Tuscany Evaluating the Impact of Updated 2013 ASCO/CAP Recommendations. Pathol Oncol Res 21(2): 477-485. 
4. French MacGrogan G, Mathieu MC Penault-Llorca F, Vincent-Salomon A Roger P, et al. (2014) Pre-analytical stage for biomarker assessment in breast cancer: 2014 update of the GEFPICS' guidelines in France. Ann Pathol 34(5): 366-372.

5. Vyberg M, Nielsen S, Røge R, Beth Sheppard, Jim Ranger Moore, et al. (2015) Immunohistochemical expression of HER2 in breast cancer: socioeconomic impact of inaccurate tests. BMC Health Serv Res 15: 352.

ISSN: 2574-1241

DOI: $10.26717 /$ BJSTR.2019.20.003454

Vincenzo Arena. Biomed J Sci \& Tech Res

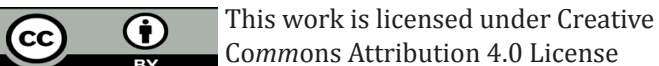

Submission Link: https://biomedres.us/submit-manuscript.php
6. Wolff AC, Hammond ME, Hicks DG, Dowsett M, McShane LM, et al. (2013) Recommendations for human epidermal growth factor receptor 2 testing in breast cancer: American Society of Clinical Oncology/College of American Pathologists clinical practice guideline update. J Clin Oncol 31(31): 3997-4013.

$\begin{array}{ll}\text { BIOMEDICAL } & \text { Assets of Publishing with us } \\ \text { RESEARCHES } & \text { - Global archiving of articles } \\ & \text { - Immediate, unrestricted online access } \\ & \text { - Rigorous Peer Review Process } \\ & \end{array}$

